\title{
An Experimental Illustration of 3D Facial Shape Analysis under Facial Expressions
}

\author{
Boulbaba Ben Amor • Hassen Drira • \\ Lahoucine Ballihi • Anuj Srivastava • \\ Mohamed Daoudi.
}

Received: date / Accepted: date

\begin{abstract}
The main goal of this paper is to illustrate a geometric analysis of 3D facial shapes in presence of varying facial expressions. This approach consists of the following two main steps: (i) Each facial surface is automatically denoised and preprocessed to result in an indexed collection of facial curves. During this step one detects the tip of the nose and defines a surface distance function with that tip as the reference point. The level curves of this distance function are the desired facial curves. (ii) Comparisons between faces are based on optimal deformations from one to another. This, in turn, is based on optimal deformations of the corresponding facial curves across surfaces under an elastic metric. The experimental results, generated using a subset of FRGC (Face Recognition Grand Challenge) v2 dataset, demonstrate the success of the proposed framework in recognizing people under different facial expressions. The recognition rates obtained here exceed those for a baseline ICP algorithm on the same dataset.
\end{abstract}

Keywords Facial shape analysis · 3D Face recognition · automatic preprocessing

Boulbaba Ben Amor

Institut Telecom ; Telecom Lille1; LIFL CNRS France.

Tel.: +33 (0) 320436418

Fax: +33 (0) 320335599

E-mail: boulbaba.benamor@telecom-lille1.eu

Hassen Drira

Institut Telecom ; Telecom Lille1; LIFL CNRS, France.

Lahoucine Ballihi

GSCM/LRIT, Faculté des Sciences, Rabat, Morocco ; LIFL CNRS, France.

Anuj Srivastava

Departement of Statistics, Florida State University, Tallahassee, FL 32306, USA.

Mohamed Daoudi

Institut Telecom ; Telecom Lille1, LIFL CNRS, France. 


\section{Introduction}

Automatic face recognition has actively been researched in recent years, and various techniques using ideas from 2D image analysis have been presented. Although a significant progress has been made, the task of automated, robust face recognition is still a distant goal. 2D Image-based methods are inherently limited by variability in imaging factors such as illumination and pose. An emerging solution is to use 3D imaging sensors for capturing surfaces of human faces, and use these data in performing face recognition. Such observations are relatively invariant to illumination and pose, although they do vary with facial expressions. As the technology for acquiring 3D facial surfaces becomes simpler and cheaper, the use of 3D facial scans will be increasingly prominent. Trying to use 3D information has become an emerging research direction in order to make face recognition more accurate and robust.

One approach is to use classical 2D images analysis techniques by forming range (depth) images from the 3D data. Hesher et al. [5] presented an efficient technique to compare certain aspects of facial shapes using ideas from image analysis: a search for optimal subspace based on stochastic gradient algorithm on a Grassman manifold was proposed. The second approach is to represent facial surfaces by certain geometrical features sets, such as the convex parts, areas with high curvatures, saddle points, etc [12]. For surface matching, the authors used classical 3D surface alignment algorithms, typically ICP (Iterative Closest Point), that compute the residual error between the probe surface and the 3D images in the gallery [1]. ICP is a procedure which iteratively minimizes the distance between two sets of points. Only a rigid transformation is taken into account in this type of algorithms. Although such feature definitions are intuitively meaningful, the computation of curvatures involves numerical approximation of second derivatives, and thus is very susceptible to observation noise. All these approaches treat the face as a rigid object and do not perform well in the presence of expression variation. However, an important requirement of a face analysis system is to handle the non-rigid of points across faces. Bronstein et al. [2] use a geodesic distance function to define level curves that are invariant to rigid motions and also to facial expressions to some extent. They consider the facial surface (only a frontal view) as an isometric surface (length preserving). Using a global transformation based on geodesics, they obtain invariant forms to facial expressions. After the transformation, they perform one classical rigid surface matching and PCA for sub-space building and face matching. The results shown were obtained on a small dataset containing 220 faces of 30 subjects - 3 artificial and 27 human. Chang et al. [3], explore an approach that matches multiple, overlapping surface patches around the nose area and then combines the results from these matches to achieve greater accuracy. This work seeks to explore what can be achieved by using a subset of the face surface that is approximately rigid across expression variation. Kakadiaris et al. [9] utilize an annotated face model to study geometrical variability across faces.

Our approach is to represent facial surfaces as indexed collections of closed curves in $\mathbb{R}^{3}$ on faces, termed facial curves, and to apply tools from shape analysis of curves. Facial curves are level curves of a surface distance function defined to be the length of the shortest path between that point and a fixed reference point (taken to be the tip of the nose) along the facial surface. This function is stable with respect to changes in facial expressions [6]. In this paper we analyze the shape of facial surfaces by analyzing the shape of facial curves. We propose an algorithm for computing geodesic paths 
between two facial surfaces and a new tool for computing geodesic distance between to finding an optimal deformations between faces.

The rest of this paper is organized as follows. In section 2, we briefly describe the FRGC dataset and the automatic data preprocessing. Section 3 describes our framework for shape analysis for facial curves and facial surfaces. In section 4, we present experiments of our framework on a subset of FRGC dataset for face recognition and authentication. Section 5 contains the concluding remarks.

\section{FRGC database description and preprocessing}

In order to assess the recognition performance of the proposed framework, we use a subset of FRGC v2 dataset. This benchmark database [15] includes 4007 3D frontal scans for 466 subjects and is considered as a challenging database as it contains sessions with both neutral and non-neutral expressions. Moreover, the laser-based 3D scanner used in the acquisition process introduces noise in the data. In fact, some of 3D face scans suffer from missing data (holes), spikes, artifacts specially in teeth region, occlusions caused by the hair, etc.

We focus in this work on designing a complete solution for 3D face analysis and recognition. For that purpose, it is crucial to begin by denoising the data by removing spikes, filling holes and extract only the useful part of face from depth image. Figure 1 shows different steps of our preprocessing solution to overcome these problems. Starting from an original range image of a face, we firstly apply a $2 \mathrm{D}$ median filter in order to remove spikes while preserving edges. Secondly, using a smooth interpolation, we fill holes by adding points in parts where the laser has been completely absorbed (e.g. eyes or eyebrows). Next, we use the Delaunay triangulation to generate a triangulated mesh from the point cloud. On this high quality mesh, we apply the procedure shown in Figure 2 to localize the nose tip, necessary for cropping the useful region of the face. For this purpose, a sphere function having center the nose tip and radius $R=100 \mathrm{~mm}$ is constructed and the part outside the sphere is cropped. Finally, a collection of geodesic level curves are extracted by locating iso-geodesic points from the reference (nose tip) using the Dijkastra algorithm [4]:

- Nose tip detection : Through the center of the mass of the face, a first transverse plane (parallel to $\mathrm{x}-\mathrm{z}$ plane) slices the facial surface. The intersection of the plane and the surface results in a horizontal profile of the face. A second sagittal plane (parallel to $\mathrm{y}-\mathrm{z}$ plane) passing through the maximum of the obtained horizontal profile, cuts the face on a vertical profile. The nose tip is located at the maximum of the middle part of this $2 \mathrm{D}$ curve as illustrated in Figure 2. This step is very important in our general framework as it is necessary to correctly crop faces and extract geodesic circles from the surface.

- Level curves extraction : Our facial shape analysis algorithm operates using 3D curves extracted by computing geodesic length function along the face. This choice is motivated by the robustness of this function to facial deformations as described in [2]. In addition to its intrinsic invariance to rigid transformations such as rotations and translations, this distance better preserves the separations of facial features as compared to the Euclidian distance in $\mathbb{R}^{3}$. 


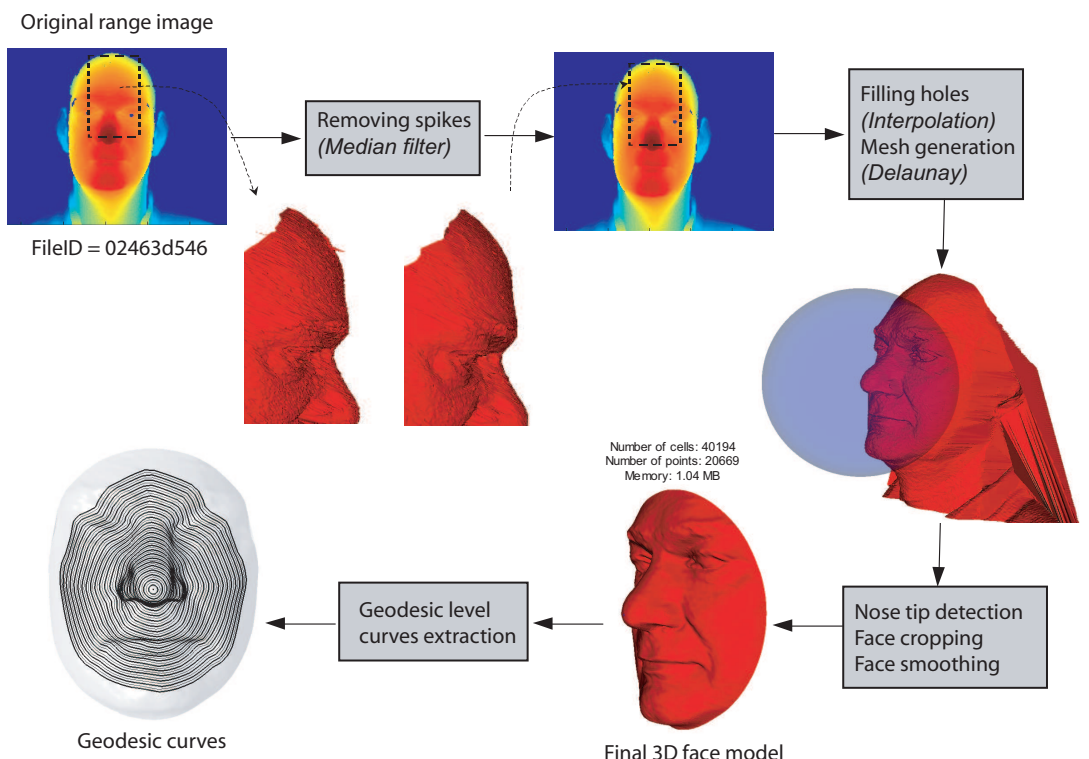

Fig. 1 Automatic FRGC data preprocessing and facial curves extraction

Let $S$ be a facial surface denoting a scanned face. Although in practice $S$ is a triangulated mesh, start the discussion by assuming that it is a continous surface. Let $D: S \longrightarrow R+$ be a continous geodesic map on $S$. Let $c_{\lambda}$ denote the level set of $D$, also called a facial curve, for the value $\lambda \in D(S)$, i.e. $c_{\lambda}=\{p \in S \mid D(P)=$ $G D(r, p)=\lambda\} \subset S$ where $r$ denotes the reference point (in our case the nose tip) and $G D(r, p)$ is the length of the shortest path from $\mathrm{r}$ to $\mathrm{p}$ on the mesh. We can reconstruct $S$ from these level curves according to $S=\cup_{\lambda} c_{\lambda}$.

The preprocessing is one of the main issues in this such problems, so we have combined the preprocessing steps described above to develop an automatic algorithm. This algorithm has successfully processed 3971 faces in FRGC v2, which means a success rate of $99.1 \%$ as described in the Table 1. This automatic preprocessing procedure failed for 16 faces taken in Fall 2003 and 20 faces taken in Spring 2004. Actually, it is the nose detection step that fails more than other steps. Figure 3 presents some examples of these faces. The main cause is the additive information which moves the mass center far from the face and the profiles extracted do no more match with the face profiles. For these faces, we have fixed manually the nose tip and so we have cleaned all the FRGC v2 faces, $99.1 \%$ automatically and $0.9 \%$ manually.

From the perspective of shape analysis, the preprocessing step is performed off-line and the timing of each preprocessing step is given in Table 2. 


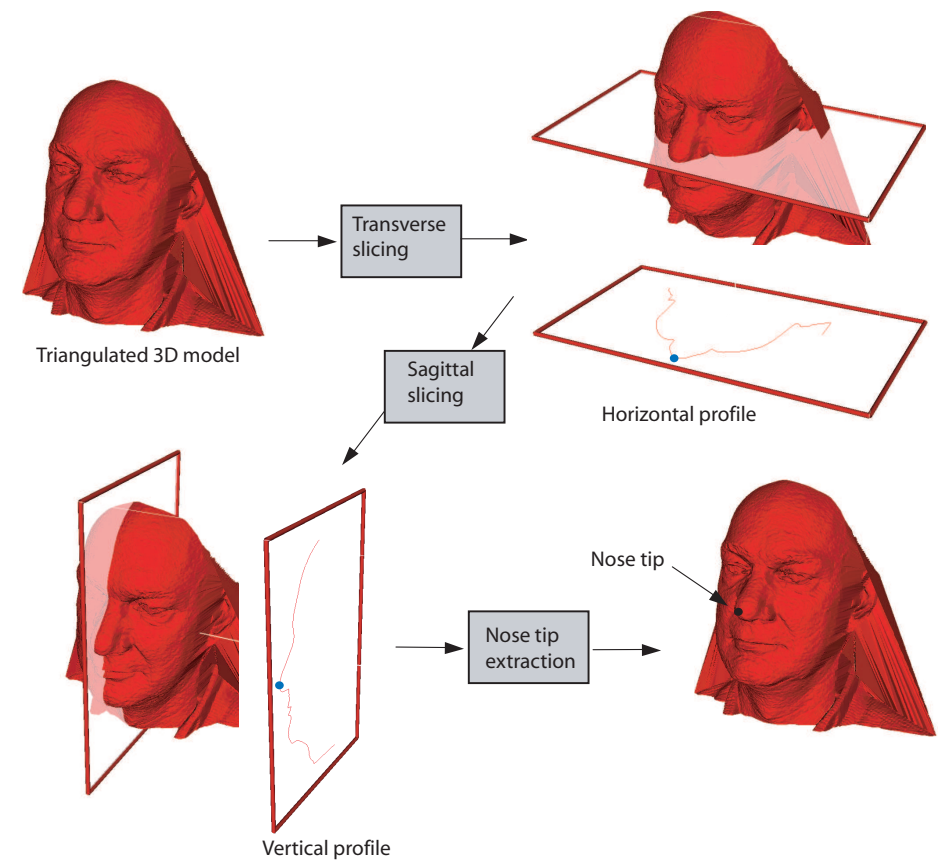

Fig. 2 Automatic nose tip detection procedure

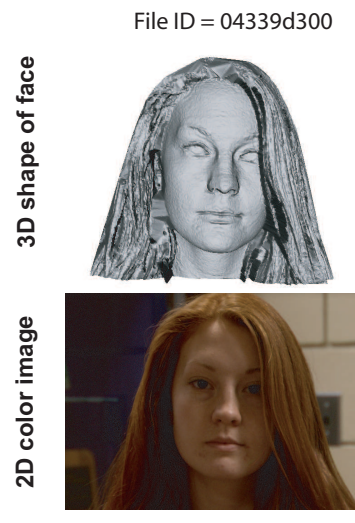

File ID $=04339 \mathrm{~d} 301$

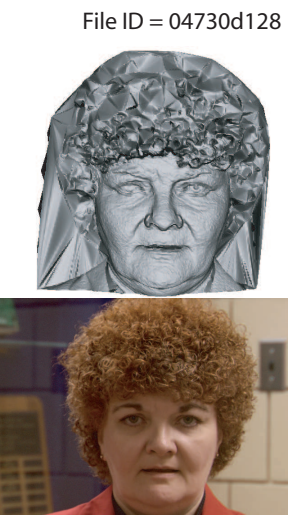

File ID $=04730 \mathrm{~d} 129$

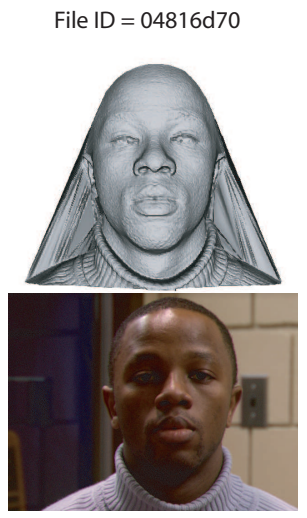

File ID $=04816 \mathrm{~d} 71$

Fig. 3 Examples of face for which preprocessing is failed

\section{A Geometric Framework for shape analysis}

As indicated earlier, our goal is to analyze shapes of facial surfaces using shapes of facial curves. In other words, we divide each surface into an indexed collection of simple, closed curves in $\mathbb{R}^{3}$ and the geometry of a surface is then studied using the geometries of the associated curves. Since these curves, previouslly called facial 
Table 1 Results of preprocessing procedure on FRGC dataset

\begin{tabular}{|c|l|l|l|l|l|}
\hline & Original files & $\begin{array}{l}\text { Success prepro- } \\
\text { cessing }\end{array}$ & $\begin{array}{l}\text { failed prepro- } \\
\text { cessing }\end{array}$ & $\begin{array}{l}\text { Success Rates } \\
(\%)\end{array}$ & \\
\hline Fall 2003 & 1893 & 1877 & 16 & 99.15 & \\
\hline Spring 2004 & 2114 & 1994 & 20 & 98.99 & \\
\hline FRGC v2 & 4007 & 3971 & 36 & 99.1 \\
\hline
\end{tabular}

Table 2 Time consuming of proprocessing steps

\begin{tabular}{|c|c|}
\hline Proprocessing step & time consuming (s) \\
\hline Median filtering & 0.091333 \\
\hline Filling holes & 3.091428 \\
\hline Delaunay triangulation & 2.578 \\
\hline Nose tipe detection & 0.093 \\
\hline Face smoothing & 1.235 \\
\hline
\end{tabular}
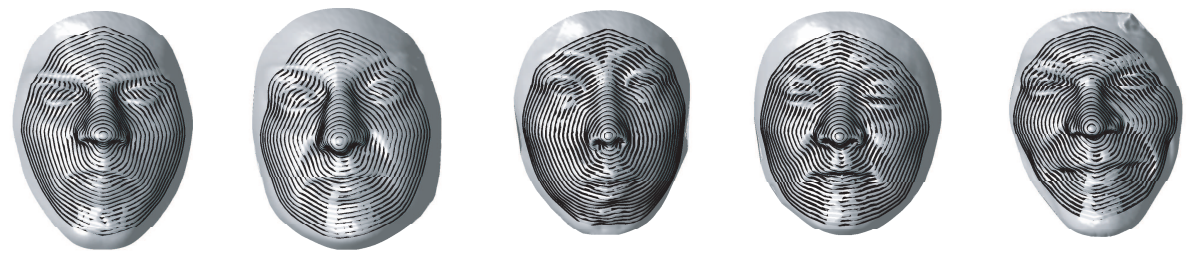

Fig. 4 Examples of face with extracted curves

curves, have been defined as level curves of an intrinsic distance function on the surface, their geometries in turn are invariant to the rigid transformation (rotation and translation) of the original surface. At least theoretically, these curves jointly contain all the information about the surface and one can go back-and-forth between the surface and the curves without any ambiguity. In practice, however, some information is lost when one works with a finite subset of these curves rather than the full set. Later, through experiments on real data, we will demonstrate that the choice of facial curves for studying shapes of facial surfaces is both natural and convenient.

In the following section, we will describe a differential-geometric approach for analyzing shapes of simple, closed curves in $\mathbb{R}^{3}$. In recent years, there have been several papers for studying shapes of continuous curves. The earlier papers, including [18, $11,13,14]$, were mainly concerned with curves in $\mathbb{R}^{2}$, while the curves in higher dimensions were studied later. In this paper, we will follow the theory laid out by Joshi et al. $[7,8]$ for elastic shape analysis of continuous, closed curves in $\mathbb{R}^{n}$ and particularize it for facial curves in $\mathbb{R}^{3}$. The mathematical framework for using elastic shape analysis of facial curves was first presented in [17].

\subsection{Facial curves}

We start by considering a closed curve $\beta$ in $\mathbb{R}^{3}$. Since it is a closed curve, it is natural to parametrize it using $\beta: \mathbb{S}^{1} \rightarrow \mathbb{R}^{3}$. We will assume that the parameterization is 
non-singular, i.e. $\|\dot{\beta}(t)\| \neq 0$ for all $t$. The norm used here is the Euclidean norm in $\mathbb{R}^{3}$. Note that the parameterization is not assumed to be arc-length; we allow a larger class of parameterizations for improved analysis. To analyze the shape of $\beta$, we shall represent it mathematically using a square-root velocity function (SRVF), denoted by $q(t)$, according to:

$$
q(t) \doteq \frac{\dot{\beta}(t)}{\sqrt{\|\dot{\beta}(t)\|}} .
$$

$q(t)$ is a special function that captures the shape of $\beta$ and is particularly convenient for shape analysis, as we describe next. Firstly, the squared $\mathbb{L}^{2}$-norm of $q$, given by:

$$
\|q\|^{2}=\int_{\mathbb{S}^{1}}\langle q(t), q(t)\rangle d t=\int_{\mathbb{S}^{1}}\|\dot{\beta}(t)\| d t
$$

which is the length of $\beta$. Therefore, the $\mathbb{L}^{2}$-norm is convenient to analyze curves of specific lengths. Secondly, as shown in [7], the classical elastic metric for comparing shapes of curves becomes the $\mathbb{L}^{2}$-metric under the SRVF representation. This point is very important as it simplifies the calculus of elastic metric to the well-known calculus of functional analysis under the $\mathbb{L}^{2}$-metric.

In order to restrict our shape analysis to closed curves, we define the set:

$$
\mathcal{C}=\left\{q: \mathbb{S}^{1} \rightarrow \mathbb{R}^{3} \mid \int_{\mathbb{S}^{1}} q(t)\|q(t)\| d t=0\right\} \subset \mathbb{L}^{2}\left(\mathbb{S}^{1}, \mathbb{R}^{3}\right)
$$

Here $\mathbb{L}^{2}\left(\mathbb{S}^{1}, \mathbb{R}^{3}\right)$ denotes the set of all functions from $\mathbb{S}^{1}$ to $\mathbb{R}^{3}$ that are square integrable. The quantity $\int_{\mathbb{S}^{1}} q(t)\|q(t)\| d t$ denotes the total displacement in $\mathbb{R}^{3}$ as one traverses along the curve from start to end. Setting it equal to zero is equivalent to having a closed curve. Therefore, $\mathcal{C}$ is the set of all closed curves in $\mathbb{R}^{3}$, each represented by its SRVF. Notice that the elements of $\mathcal{C}$ are allowed to have different lengths. Due to a nonlinear (closure) constraint on its elements, $\mathcal{C}$ is a nonlinear manifold. We can make it a Riemannian manifold by using the metric: for any $u, v \in T_{q}(\mathcal{C})$, we define:

$$
\langle u, v\rangle=\int_{\mathbb{S}^{1}}\langle u(t), v(t)\rangle d t .
$$

We have used the same notation for the Riemannian metric on $\mathcal{C}$ and the Euclidean metric in $\mathbb{R}^{3}$ hoping that the difference is made clear by the context. For instance, the metric on the left side is in $\mathcal{C}$ while the metric inside the integral on the right side is in $\mathbb{R}^{3}$. For any $q \in \mathcal{C}$, the tangent space:

$$
T_{q}(\mathcal{C})=\left\{v: \mathbb{S}^{1} \rightarrow \mathbb{R}^{3} \mid\langle v, w\rangle=0, w \in N_{q}(\mathcal{C})\right\},
$$

where $N_{q}(\mathcal{C})$, the space of normals at $q$ is given by:

$N_{q}(\mathcal{C})=\operatorname{span}\left\{\frac{q^{1}(t)}{\|q(t)\|} q(t)+\|q(t)\| \mathbf{e}^{1}, \frac{q^{2}(t)}{\|q(t)\|} q(t)+\|q(t)\| \mathbf{e}^{2}, \frac{q^{3}(t)}{\|q(t)\|} q(t)+\|q(t)\| \mathbf{e}^{3}\right\}$,

and where $\left\{\mathbf{e}^{1}, \mathbf{e}^{2}, \mathbf{e}^{3}\right\}$ form an orthonormal basis of $\mathbb{R}^{3}$.

So far we have described a set of closed curves and have endowed it with a Riemannian structure. Next we consider the issue of representing the shapes of these curves. It is easy to see that several elements of $\mathcal{C}$ can represent curves with the 
same shape. For example, if we rotate a curve in $\mathbb{R}^{3}$, we get a different SRVF but its shape remains unchanged. Another similar situation arises when a curve is reparameterized; a re-parameterization changes the SRVF of curve but not its shape. In order to handle this variability, we define orbits of the rotation group $S O(3)$ and the re-parameterization group $\Gamma$ as the equivalence classes in $\mathcal{C}$. Here, $\Gamma$ is the set of all orientation-preserving diffeomorphisms of $\mathbb{S}^{1}$ (to itself) and the elements of $\Gamma$ are viewed as re-parameterization functions. For example, for a curve $\beta: \mathbb{S}^{1} \rightarrow \mathbb{R}^{3}$ and a function $\gamma: \mathbb{S}^{1} \rightarrow \mathbb{S}^{1}, \gamma \in \Gamma$, the curve $\beta(\gamma)$ is a re-parameterization of $\beta$. The corresponding SRVF changes according to $q(t) \mapsto \sqrt{\dot{\gamma}(t)} q(\gamma(t))$. We set the elements of the set:

$$
[q]=\{\sqrt{\dot{\gamma}(t)} O q(\gamma(t)) \mid O \in S O(3), \quad \gamma \in \Gamma\},
$$

to be equivalent from the perspective of shape analysis. The set of such equivalence classes, denoted by $\mathcal{S} \doteq \mathcal{C} /(S O(3) \times \Gamma)$ is called the shape space of closed curves in $\mathbb{R}^{3}$. $\mathcal{S}$ inherits a Riemannian metric from the larger space $\mathcal{C}$ and is thus a Riemannian manifold itself.

The main ingredient in comparing and analyzing shapes of curves is the construction of a geodesic between any two elements of $\mathcal{S}$, under the Riemannian metric given in Eqn. 3. Given any two curves $\beta_{1}$ and $\beta_{2}$, represented by their SVRFs $q_{1}$ and $q_{2}$, we want to compute a geodesic path between the orbits $\left[q_{1}\right]$ and $\left[q_{2}\right]$ in the shape space $\mathcal{S}$. This task is accomplished using a path straightening approach which was introduced in [10]. The basic idea here is to connect the two points $\left[q_{1}\right]$ and $\left[q_{2}\right]$ by an arbitrary initial path $\alpha$ and to iteratively update this path using the negative gradient of an energy function $E[\alpha]=\frac{1}{2} \int_{s}\langle\dot{\alpha}(s), \dot{\alpha}(s)\rangle d s$. The interesting part is that the gradient of $E$ has been derived analytically and can be used directly for updating $\alpha$. As shown in [10], the critical points of $E$ are actually geodesic paths in $\mathcal{S}$. Thus, this gradient-based update leads to a critical point of $E$ which, in turn, is a geodesic path between the given points.

Figure 5 shows some illustrations of this idea. The top row two facial surfaces and five level curves extracted from each of these surfaces. The remaining five rows display geodesic paths between the corresponding level curves of the two faces, obtained using the path-straightening approach. In each case, the first and the last curves are the ones extracted from the two surfaces, and the intermediate curves denote equally-spaced points on the corresponding geodesic $\alpha$. These curves have been scaled to the same length to improve display of geodesics. We will use the notation $d\left(\beta_{1}, \beta_{2}\right)$ to denote the geodesic distance, or the length of the geodesic in $\mathcal{S}$, between the two curves $\beta_{1}$ and $\beta_{2}$.

Why do we expect that shapes of facial curves are central to analyzing the shapes of facial surfaces? There is plenty of psychological evidence that certain facial curves, especially those around nose, lips and other prominent parts, can capture the essential features of a face. Our experiments support this idea in a mathematical way. We have computed geodesic distances between corresponding facial curves of different faces - same people different facial expressions and different people altogether. We have found that the distances are typically smaller for faces of the same people, despite different expressions, when compared to the distances between facial curves of different people. Shown in Figure 6 is an example of this experiment. In the top row, we show faces and facial curves of three faces, the first two belonging to one person and the last belonging to another person. In the bottom left of the figure, we plot the geodesic distance $d\left(c_{\lambda}^{1}, c_{\lambda}^{2}\right)$ versus $\lambda$ for two cases: The first case is for 


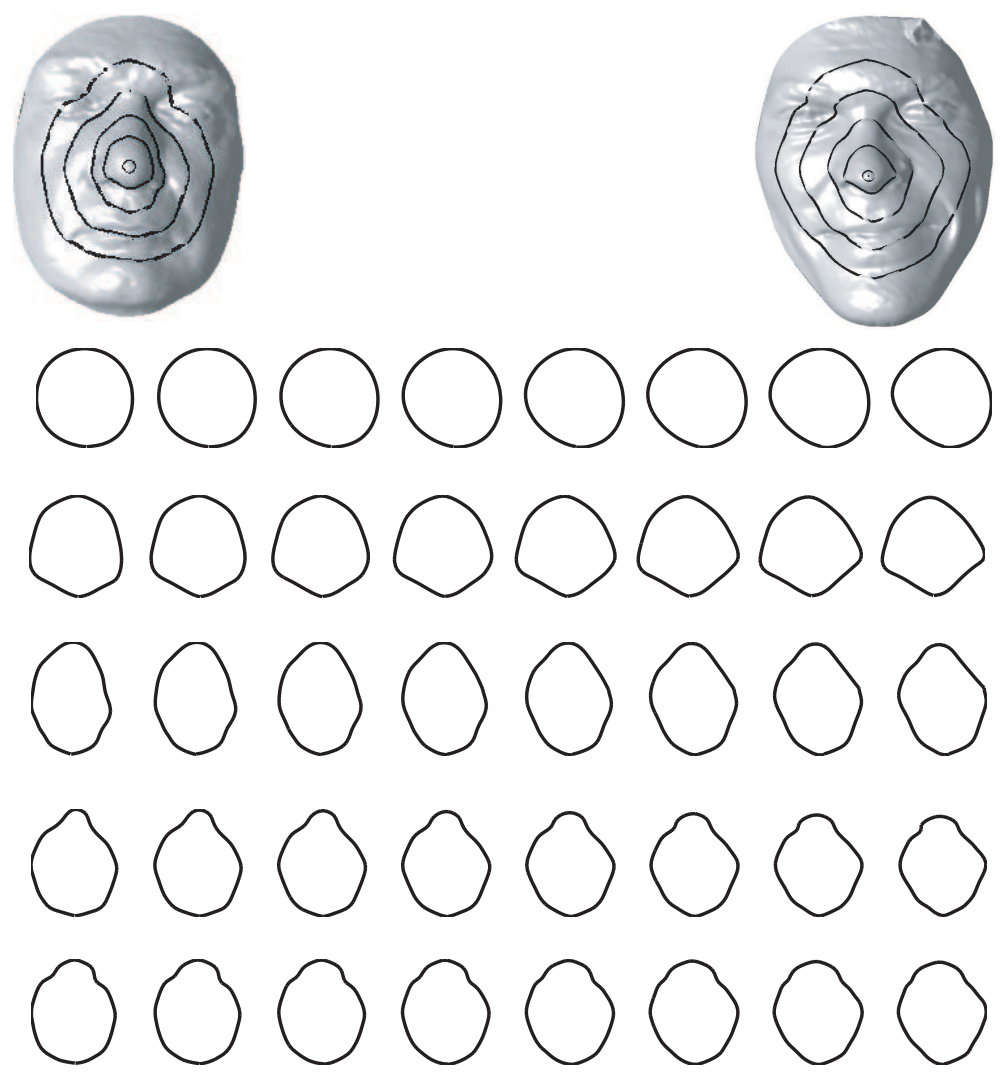

Fig. 5 Examples of geodesic between curves

facial curves from the first two faces, i.e. same person, and the distance is shown using a broken line. The second case is for facial curves from different people (first and the last face in the first row) and the distance is shown using the solid line. As this experiment suggest, the differences in shapes of facial curves is generally smaller for the same person and larger for different persons. Therefore, shapes of facial curves can play a central role in analyzing shapes of facial surfaces.

\subsection{Facial surfaces}

Now we extend ideas developed in the previous section for analyzing shapes of facial curves to the shapes of full facial surfaces. As mentioned earlier, we are going to represent a facial surface $S$ with an indexed collection of the level curves of the $D$ function. That is,

$$
S \leftrightarrow\left\{c_{\lambda}, \lambda \in[0, L]\right\},
$$

where $c_{\lambda}$ is the level set associated with $D=\lambda$. Through this relation, each facial surface has been represented as an element of the set $\mathcal{C}^{[0, L]}$. In our framework, the shapes of any two faces are compared by comparing their corresponding facial curves. Given any two surfaces $S^{1}$ and $S^{2}$, and their facial curves $\left\{c_{\lambda}^{1}, \lambda \in[0, L]\right\}$ 


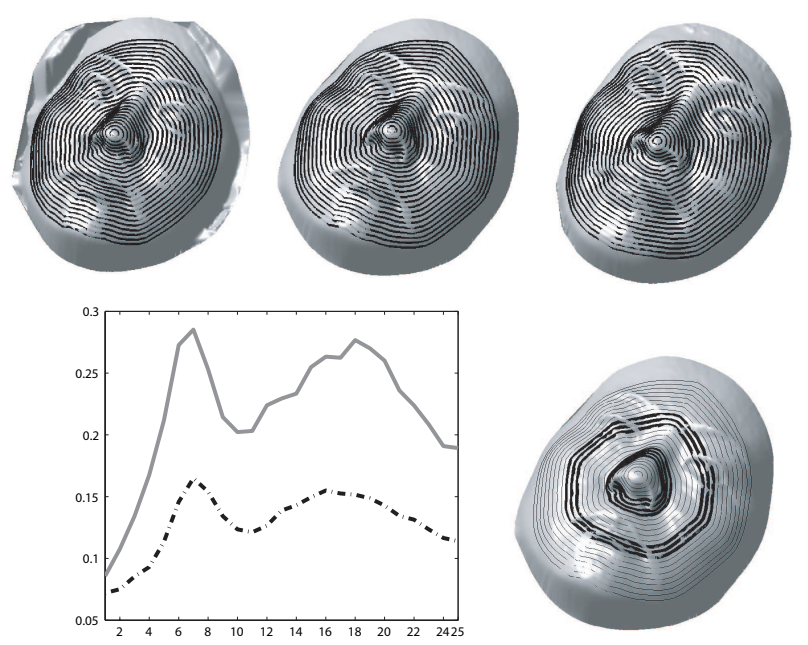

Fig. 6 Top rows shows facial curves extracted from three example facial surfaces - the first two belong to the same person and the last belongs to another person. The bottom left shows the plot of the mean of distances for each level $d\left(c_{\lambda}^{1}, c_{\lambda}^{2}\right)$ for two cases - for 125 genuine accesses (broken line) and for 125 impostor accesses (solid line). The bottom right highlights the two regions - one around the nose profile and the other containing eyes and mouth - that have higher values in the distance plot.

and $\left\{c_{\lambda}^{2}, \lambda \in[0, L]\right\}$, respectively, our idea is to compare the facial curves $c_{\lambda}^{1}$ and $c_{\lambda}^{2}$, and to accumulate these differences over all $\lambda$. More formally, we define a distance: $d_{s}: \mathcal{C}^{[0, L]} \times \mathcal{C}^{[0, L]} \rightarrow \mathbb{R}_{\geq 0}$, given by

$$
d_{s}\left(S^{1}, S^{2}\right)=\int_{0}^{L} d\left(c_{\lambda}^{1}, c_{\lambda}^{2}\right) d \lambda .
$$

Here, the distance inside the integral is the geodesic distance function between the shapes of any curves, described in the last section. It has been shown in [16] that this distance is actually a proper distance between the elements of the $\mathcal{C}^{[0, L]}$.

In addition to the distance $d_{s}\left(S^{1}, S^{2}\right)$, which is useful in biometry and other classification experiments, we also have a geodesic path in $\mathcal{C}^{[0, L]}$ between the two points representing by $S^{1}$ and $S^{2}$. This geodesic corresponds to the optimal elastic deformations of facial curves and, thus, facial surfaces from one to other. Shown in Figure 7 are examples of such geodesic paths between given facial surfaces - some examples involves faces of same people but with different facial expressions while the other examples show geodesics between faces that belong to different people.

\section{Experiments}

Our framework is designed for computing geodesic path between facial shapes via the shape of their curves and the geodesic distance $d_{s}$ is used as the similarity score. Shown in Figure 8 are three examples of similarity scores between faces in different situations. Each of the three matrices in that figure shows similarity scores between $15 \times 15$ faces. The first case involves neutral expressions on the column and different 
(a)
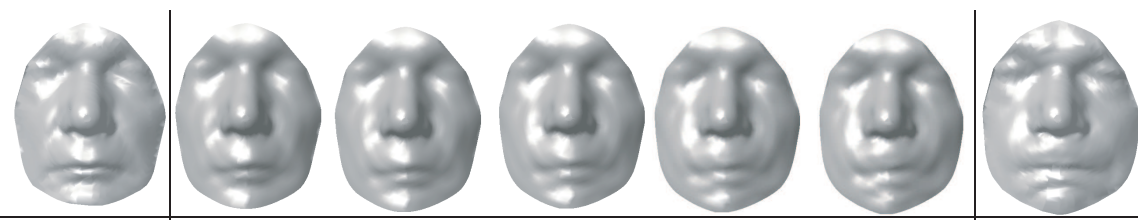

(b)
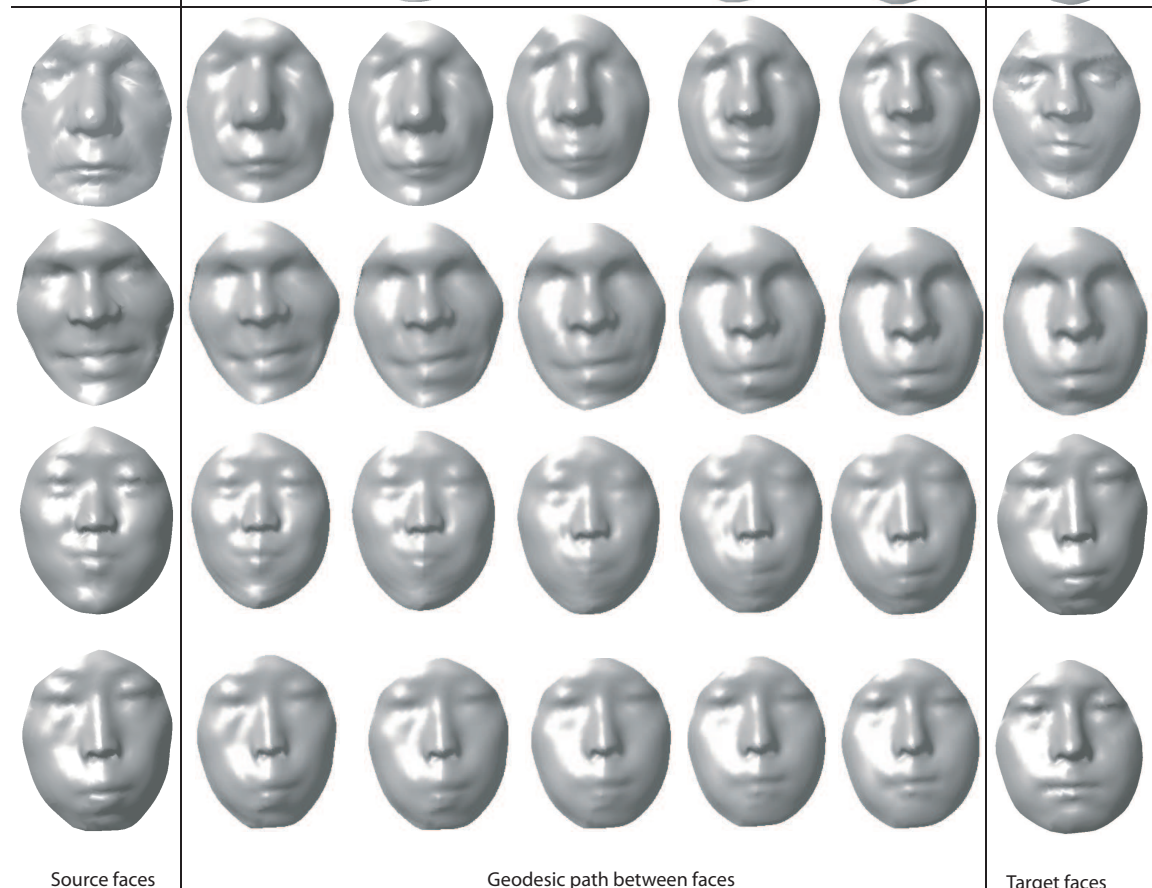

Geodesic path between faces
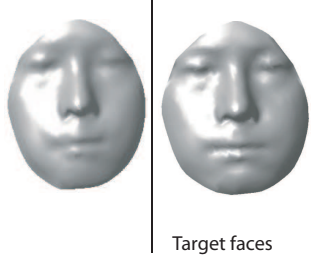

Fig. 7 Geodesic path between source and target faces (a) intra-class path, source and target with different expressions (b) inter-class path

neutral expressions on the row, but of the same 15 people. The faces have been arranged in the same order so that the scores between the same person lies long the diagonal. As expected, the values along the diagonal are in general much smaller (darker in the image) than the non-diagonal values. The remaining two matrices are similar except that the faces along the rows now have small expression variation (middle image) and large expression variation (right image).

One interesting target application of our framework is face authentication and recognition.

In this section, we present some experimental evaluations produced on a subset of FRGC v2 database. We note that the main challenge in $3 \mathrm{D}$ face recognition is to handle deformations of faces caused by expressions or emotions.

- Evaluation protocol: we focus in our experiments on the common biometric scenarions which are authentication (or verification) and identification (or recognition). One application of the verification task is access control where an authorized individual is seeking access to a secure facility and presents to the system his or her identity. Here, a one-to-one matching is performed : the 3D image for this 


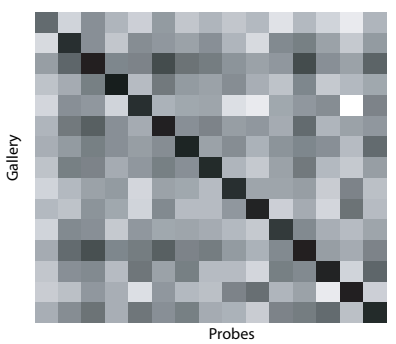

(a) Neutral vs. neutral expressions similarity matrix (inter-sessions)

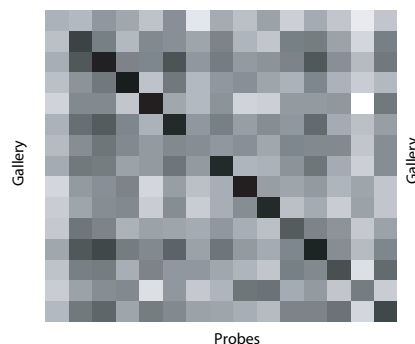

(b) Neutral vs. small expressions similarity matrix (inter-sessions)

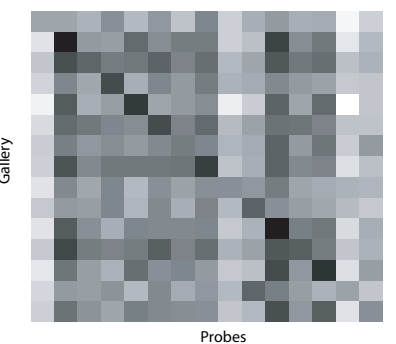

(c) Neutral vs. large expressions similarity matrix (inter-sessions)

Fig. 8 Similarity matrices for (a) neutral vs. neutral comparsions, (b) neutral vs. small expressions, and (c) neutral vs. large expressions

individual is acquired, preprocessed and finally compared to an enrollment acquisition already incorporated in the system database. If the similarity is greater than a defined threshold the subject is granted access, otherwise access is denied. The identification scenarion consists on determining identity of an authorized user in a database of many individuals.

As far as verification scenario is concerned, it is important to examine the probability of correct verification as a function of the false acceptance rate (or imposter access). This is often shown with a Receiver Operating Characteristic (ROC) curve that graphs the probability of correct verification versus the false acceptance rate. In the identification scenario, however, the results for facial identification are often displayed using a Cumulative Match Characteristic (CMC) curve. This curve displays the cumulative identification rates as a function of the rank distribution. This provides an indication of how close one may be to getting the correct match if the rank-one match was incorrect.
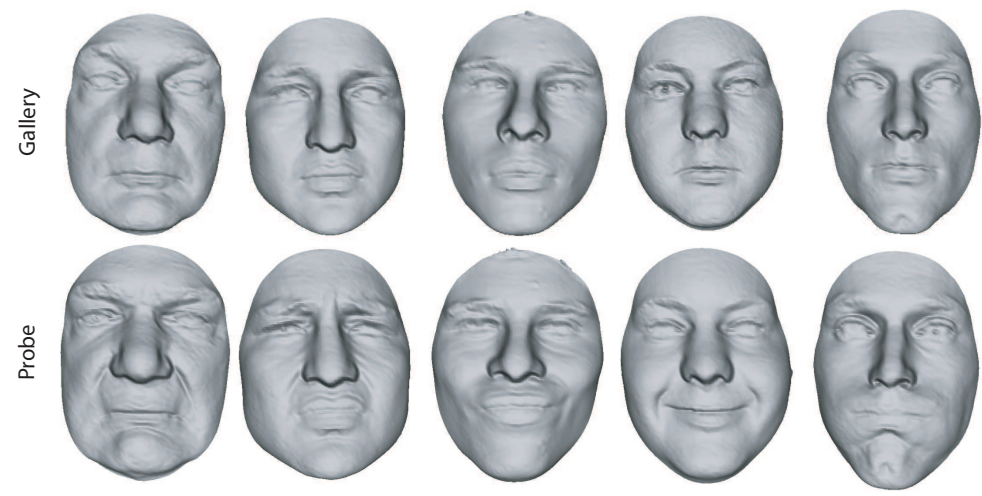

Fig. 9 Sample faces from gallery (heigher row) and probe datasets (lower row)

In order to produce results for both scenarios and to explore effect of the presence of facial expressions on performance, a similarity matrix between a gallery and a probe dataset is computed. The gallery dataset contains 125 sessions for 125 dif- 
ferent subjects acquired with neutral expressions selected from FRGC v2 dataset. The probe dataset includes completely different sessions of these subjects under non-neutral facial expressions, as shown in Figure 9. Due to sensitivity of our algorithm to opened mouth, expressions in probe dataset include only scans with closed mouths.

In this matrix, the diagonal terms represent match scores (or Genuine Access) contrary to non-diagonal terms which represent Non-match scores (or Imposter Access). These scores allow us to produce the ROC and the CMC curves for this scenario.

- Preliminary results : We compare results of our algorithm with a standard implementation of ICP which is considered as a baseline in 3D face recognition. The same protocol was followed to compute similarity matrices for both the algorithms on the same preprocessed data.

As shown in Figure 10 the ROC curve of our approach is always above the ICP one which means that our verification rate at each false accept rate is greater than ICP verification rate. In addition, the Equal Error Rate which is the error rate for false accept rate equal to false reject rate, is lower in our case $(8 \%)$ than the ICP case $(15 \%)$.

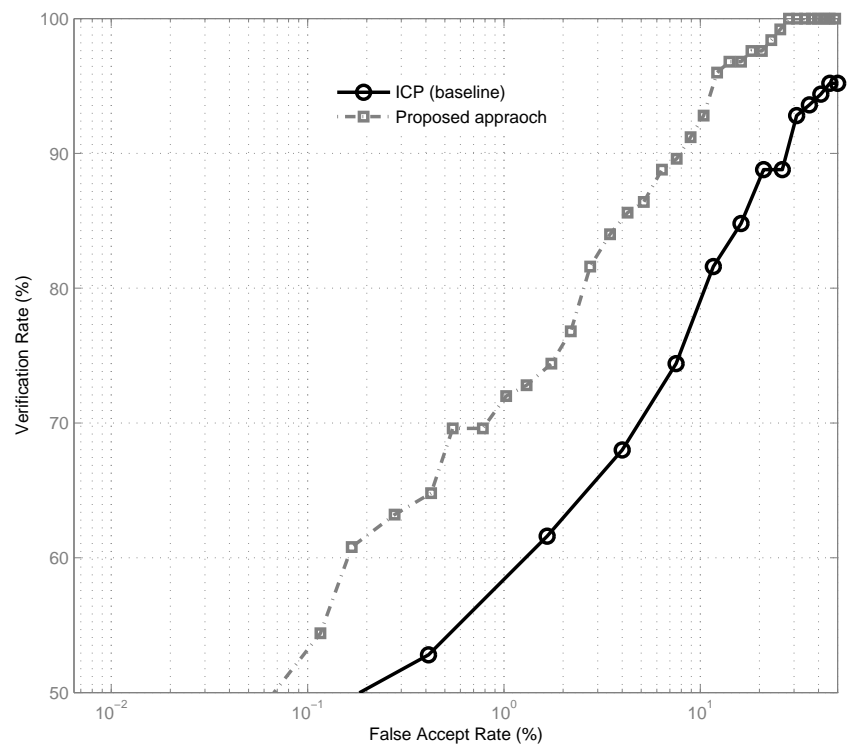

Fig. 10 Receiver operating characteristic curves for our approach and ICP (baseline)

These results are confirmed with the CMC curves for the two algorithms (Figure 11). In fact, rank-one recognition rate given by our algorithm is about $88.8 \%$ compared to $78.5 \%$ given by the ICP algorithm. Moreover, at the fourth rank, our 
algorithm is already able to recognize $97.8 \%$ of the subjects in contrast with ICP which recognition rate at this rank is still under $89 \%$.

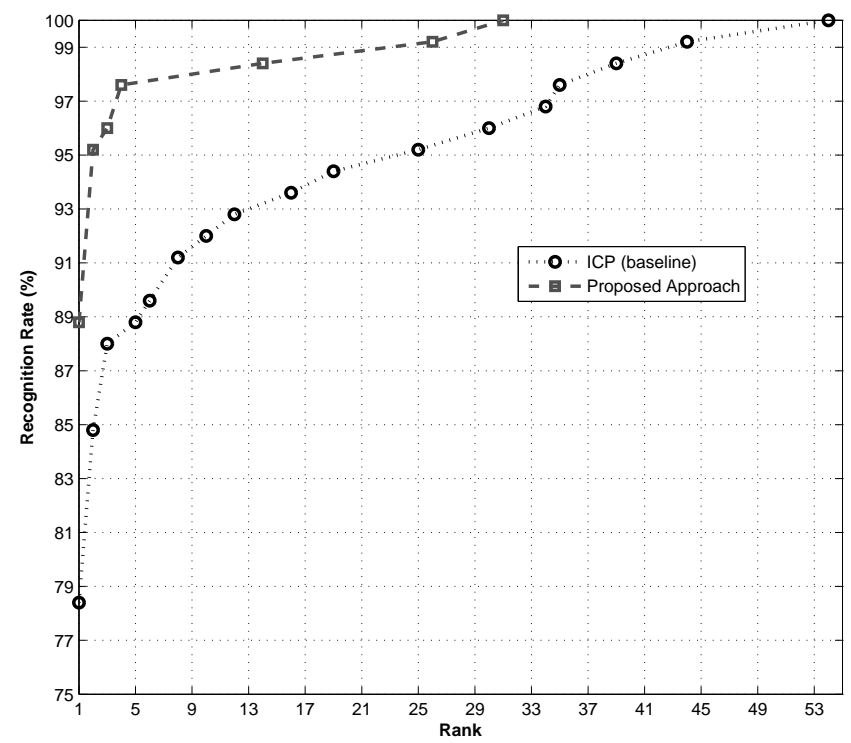

Fig. 11 Cumulative Match Characteristic curves for our approach and ICP (baseline)

\section{Conclusions}

In this paper, we have illustrated a geometric analysis of 3D facial shapes in presence of both netural and non-neutral facial expressions. In this analysis, the preprocessing is completely automated - the algorithm processes the face scan data, detects the tip of the nose and extracts a set of facial curves. The main tool presented in this paper is the construction of geodesic paths between arbitrary two facial surfaces. The length of a geodesic between any two facial surfaces is computed as the geodesic length between a set of their facial curves. This length quantifies differences in their shapes ; it also provides an optimal deformation from one to the other. In order to validate our approach in presence of facial expressions, a similarity matrix between 125 probe images with facial expressions and 125 gallery images with the neutral expression is computed. Authentication and recognition scores are produced and compared with a standard implementation of ICP as a baseline. The results of our algorithm outperform the baseline ICP algorithm which prove robustness of the proposed framework to deformations caused by facial expressions.

Our approach extract closed curves from the face using geodesic distance and one reference point (the nose tip). Curve extraction procedure is not adapted to the opened 

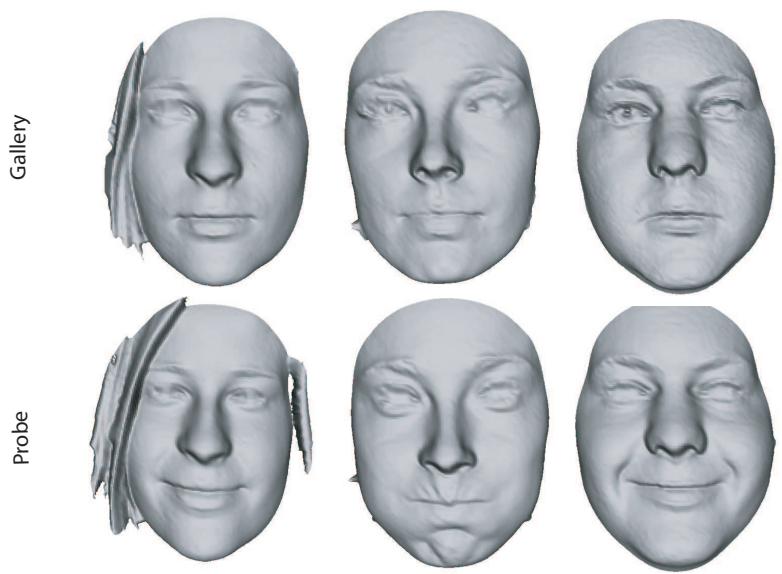

Fig. 12 Examples of difficult faces not recognized by our algorithm

mouth problem. In order to adapt it to solve this problem we are working on automatically localizes and then remove mouth region for all faces (neutral and non-neutral). We plan to fill the hole with an interpolated mesh in which geodesic path will be forced to zero. To compute geodesic path from the reference point to another point under the mouth, the filled hole is used to reconstruct the path between the two points but geodesic distance inside is nil. Therefore, none of the level curves will pass through the mouth region. This procedure requires defining distances on facial surfaces using different metrics for mouth and non-mouth regions and will be implemented in our future research.

\section{References}

1. B. Ben Amor, M. Ardabilian, and L. Chen. New experiments on icp-based 3d face recognition and authentication. In ICPR '06: Proceedings of the 18th International Conference on Pattern Recognition, pages 1195-1199, 2006.

2. A. M. Bronstein, M.M. Bronstein, and R. Kimmel. Three-dimensional face recognition. International Journal of Computer Vision, 64(1):5-30, 2005.

3. K. I. Chang, K. W. Bowyer, and P. J. Flynn. Multiple nose region matching for 3D face recognition under varying facial expression. IEEE Transactions on Pattern Analysis and Machine Intelligence, 28(10):1695-1700, 2006.

4. Edsger Wybe Dijkstra. A note on two problems in connection with graphs. Numerische Math., 1:269-271, 1959 .

5. C. Hesher, A. Srivastava, and G. Erlebacher. A novel technique for recognizing faces using range images. In Proceedings of ISSPA, 2003, Paris, France, 2003.

6. M. Hilaga, S. T. Kohmura, and T. L. Kunii. Topology matching for fully automatic similarity estimation of 3d shapes. In $A C M$ SIGGRAPH, Annual Conference Series, page 203212, 2001.

7. S. H. Joshi, E. Klassen, A. Srivastava, and I. H. Jermyn. A novel representation for efficient computation of geodesics between $n$-dimensional curves. In IEEE CVPR, 2007.

8. S. H. Joshi, E. Klassen, A. Srivastava, and I. H. Jermyn. Removing shape-preserving transformations in square-root elastic (SRE) framework for shape analysis of curves. In A. Yuille et al., editor, EMMCVPR, LNCS 4679, pages 387-398, 2007. 
9. I. A. Kakadiaris, G. Passalis, G. Toderici, M. N. Murtuza, N. Karampatziakis, and T. Theoharis. Three-dimensional face recognition in the presence of facial expressions: An annotated deformable model approach. IEEE Transactions on Pattern Analysis and Machine Intelligence, 29(4):1-10, 2007.

10. E. Klassen and A. Srivastava. Geodesics between 3D closed curves using pathstraightening. In Proceedings of ECCV, Lecture Notes in Computer Science, pages I: 95-106, 2006

11. E. Klassen, A. Srivastava, W. Mio, and S. Joshi. Analysis of planar shapes using geodesic paths on shape spaces. IEEE Pattern Analysis and Machine Intelligence, 26(3):372-383, March, 2004.

12. X. Lu, A. K. Jain, and D. Colbry. Matching $2.5 \mathrm{~d}$ face scans to $3 \mathrm{~d}$ models. IEEE Transactions on Pattern Analysis and Machine Intelligence, 28(1):31-43, Jan. 2006.

13. P. W. Michor and D. Mumford. Riemannian geometries on spaces of plane curves. Journal of the European Mathematical Society, 8:1-48, 2006.

14. W. Mio, A. Srivastava, and S. Joshi. On shape of plane elastic curves. International Journal of Computer Vision, 73(3):307-324, 2007.

15. P. Jonathon Phillips, Patrick J. Flynn, Todd Scruggs, Kevin W. Bowyer, Jin Chang, Kevin Hoffman, Joe Marques, Jaesik Min, and William Worek. Overview of the face recognition grand challenge. In CVPR '05: Proceedings of the 2005 IEEE Computer Society Conference on Computer Vision and Pattern Recognition (CVPR'05) - Volume 1, pages 947-954, Washington, DC, USA, 2005. IEEE Computer Society.

16. C. Samir, A. Srivastava, M. Daoudi, and E. Klassen.

17. A. Srivastava, C. Samir, S. Joshi, and M. Daoudi. Elastic shape models for face analysis using curvilinear coordinates. Journal of Mathematical Imaging and Vision, accepted for publication, 2008.

18. L. Younes. Computable elastic distance between shapes. SIAM Journal of Applied Mathematics, 58:565-586, 1998. 